\title{
PARALLEL OPTION PRICE VALUATIONS WITH THE EXPLICIT FINITE DIFFERENCE METHOD*
}

\author{
ALEXANDROS V. GERBESSIOTIS ${ }^{\dagger}$
}

\begin{abstract}
We show how computations such as those involved in American or European-style option price valuations with the explicit finite difference method can be performed in parallel. Towards this we introduce a latency tolerant parallel algorithm for performing such computations efficiently that achieves optimal theoretical speedup $p$, where $p$ is the number of processor of the parallel system. An implementation of the parallel algorithm has been undertaken, and an evaluation of its performance is carried out by performing an experimental study on a high-latency PC cluster, and at a smaller scale, on a multi-core processor using in addition the SWARM parallel computing framework for multi-core processors. Our implementation of the parallel algorithm is not only architecture but also communication library independent: the same code works under LAM-MPI and Open MPI and also BSPlib, two sets of library frameworks that facilitate parallel programming. The suitability of our approach to multi-core processors is also established.
\end{abstract}

Key words. parallel computing, parallel algorithm, parallel programming, BSP model, performance analysis, explicit finite difference method, option valuations, latency tolerant algorithms

AMS subject classifications. 68W10, 68Q25, 68W40, 65-05

1. Introduction. We show how option price valuations of assets such as Americanstyle stock options can be performed in parallel by using the explicit finite difference method (EFDM). We present a latency tolerant parallel algorithm for performing these valuations in optimal parallel time, thus achieving optimal parallel speedup. Although the parallelization of the corresponding sequential algorithm into an optimal latency-tolerant parallel algorithm is not complex, it involves a transformation of the standard representation of a trinomial tree to facilitate the blocked structure of the parallel operations in a latency-tolerant and computation and communication optimal way. We use the Bulk-Synchronous Parallel (BSP) model of computation to design and analyze the proposed algorithm in an architecture independent way. We confirm the theoretical conclusions drawn from the analysis of the proposed algorithm by performing an experimental study of an implementation of the proposed algorithm on a high-latency parallel platform consisting of a cluster of PC workstations connected by a high performance ethernet switch. The experimental results obtained seem to validate the usefulness and usability of the BSP model in measuring parallel computation and communication performance. We have implemented the latency tolerant code to work without modifications and only through a recompilation under BSPlib [14], LAM-MPI [15], Open MPI [11] on clusters of PC workstations and also multi-core processors. In addition, through modifications, our algorithm implementation can also run on dual-core processors under SWARM [1], a parallel computing framework for multi-core processors.

The hardware abstraction of the BSP model that is used for algorithm design and analysis in this work consists of three components: (a) a collection of $p$ processor/memory components numbered $0, \ldots, p-1,(\mathrm{~b})$ a communication network that can deliver messages point to point among the components, and (c) a mechanism for efficient synchronization, in barrier style, of all or a subset of the components. The

\footnotetext{
${ }^{*}$ This work was supported in part by NSF/MRI NSF-9977508 and NSF/ITR IIS-0324816 grants.

${ }^{\dagger}$ CS Department, New Jersey Institute of Technology, Newark, NJ 07102, USA. (alexg@cs.njit.edu).
} 
performance of any BSP computer can be characterized by: (a) the number of components $p$, (b) the minimum elapsed time $L$, measured in basic computational operations, between successive synchronization operations, and (c) - the cost $g$ of communication per word (inverse of router throughput). Computation on the BSP model proceeds in a succession of supersteps. Each superstep $S$ is charged $\max \{L, x+g h\}$ basic time steps, where $x$ is the maximum number of basic computational operations executed by any processor during $S$, and $h$ is the maximum number of messages transmitted or received by any processor. We specify the performance of a BSP algorithm $\mathcal{A}$ by comparing its performance to a sequential algorithm $\mathcal{A}^{*}$. We then specify two ratios $\pi$ and $\mu ; \pi$, is the ratio $\pi=p C_{\mathcal{A}} / C_{\mathcal{A}^{*}}$ and $\mu$, is the ratio $\mu=p M_{\mathcal{A}} / C_{\mathcal{A}^{*}}$, where $C_{\mathcal{A}}, M_{\mathcal{A}}$ are the computation and communication time of $\mathcal{A}$ and $C_{\mathcal{A}^{*}}$ is the computation time of $\mathcal{A}^{*}$. Finally, conditions on $n, p, L$ and $g$ are derived that are sufficient for the algorithm to make sense and the claimed bounds on $\pi$ and $\mu$ to hold. Some sufficient conditions for the most interesting optimality criteria, such as $c$-optimality, i.e., $\pi=c+o(1)$ and $\mu=o(1)$ can also be claimed in the form of corollaries. All asymptotic bounds refer to the problem size as $n \rightarrow \infty$.

1.1. Contributions and previous work. The results presented in this work supplement the latency-tolerant BSP-oriented algorithms for option price valuations on binomial trees [7] and trinomial trees [8]. Recently there has been a surge of interest in developing and studying the performance of parallel algorithms for option price valuations. Intel [13] published a white paper in which they investigate the performance of binomial tree valuations on multi-core processors. The approach of performing option price valuations using the explicit finite difference method used in this work is equivalent to performing discounted expectations in a trinomial tree [5].

Although the proposed algorithms has been developed as a latency-tolerant algorithm that can be supported by and exhibit high efficiency if run on a cluster of PC workstations, it is also portable, efficient and adaptable enough to work on multi-core processors. Even though the work of [13] discusses option pricing using the BlackScholes formula or binomial trees, the benefit of using trinomial trees or the equivalent explicit finite difference method has not yet been adequately explored in that setting. Our generic latency-tolerant pc-cluster oriented algorithm can easily work efficiently on multi-core processors as well.

The approach of using the explicit finite difference method for option pricing as presented in this work has some benefits over a direct trinomial tree approach used in [8]. It further minimizes communication and synchronization time thus reducing latency-based overhead, than by simply using blocking $[8,7]$ techniques. For example, in Theorem 3.1 that will be established in this work, communication contributions are approximately $2 L n / b+2 g n$, where $p, L, g$ are the BSP parameters, $n$ is problem size, and $b$ is a blocking factor parameter inherent in the algorithm. This is significantly less compared to [8], where communication contributes approximately $3 L n / b+g n^{2} / p b+g n$. As long as $n \gg p b$ our approach is more communication efficient than the one in [8] in addition to being also more synchronization effective. The condition $n \gg p b$ is necessary for the introduced algorithm to work properly anyway, and this guarantees communication efficiency. For the algorithm in [8] the condition $n \gg p b$ is necessary for that algorithm to achieve one-optimality in computation at the expense of minimizing communication. This is because in the algorithm introduced in this paper sequential time and consequently parallel time of approximately $2 n^{2}$ and $2 n^{2} / p+n$ are achieved. The algorithm in [8] requires approximately $n^{2}$ and $n^{2} / p+n b$ respectively. 
The experiments presented in [8] that generate interesting speedup results are for large $n=32768,65536$ whereas the ones in this work work even for $n=8192$. Even though for for these large values of $n$ the condition $n \gg p b$ might be true, this is not always so. This means that $n^{2} / p+n b$ might be much larger than $2 n^{2} / p$. For example in [8] for $n=32758, p=16$ and $b=1024$ the running time of the trinomial tree algorithm is around 21.55 seconds, whereas in this work, Table 4.1 gives a time of around 21.27 seconds if one divides the sequential time indicated by the corresponding speedup figure. The other figures for $n=32768$ are comparable up to variations of 5 10\%. Furthermore, in Table 4.6 for $p=32$, running times under MPI of 14.79 to 16.97 are achieved for $b=1025$ and $b=513$ which are significantly less than those reported in [8] which range from 17.79 to 22.23 for $b=1024$ and $b=512$ respectively there. Thus this is an experimental confirmation of the theoretical analysis of the previous paragraph. In additional scalable results in this work are available for $n=8192$ and $n=16384$, whereas [8] only reports results for $n=32768,65536$.

2. Option price valuations: Trinomial trees and EFDM. Option pricing under the Black-Scholes model [3] requires the solution of the partial differential equation satisfied by the option price and can be obtained by building a discrete time and state binomial model of the asset price and then applying discounted expectations [5]. A generalization of this model [17] to a trinomial model is also useful. The solution of the partial differential equation of the option price by the explicit finite difference method [16] is also equivalent to performing discounted expectations in a trinomialtree [5]. Trinomial trees are advantageous over binomial trees because the asset price in a trinomial-tree moves in three directions compared with only two for a binomial tree and thus the time horizon (number of time steps in the future) of the former can be reduced to achieve the accuracy of the latter. There are various ways one can choose the jump size and move probabilities of a trinomial-tree. For an asset whose current price is $S$, and $r$ is the riskless and continuously compounded interest rate, the stochastic differential equation for the risk-neutral geometric Brownian motion (GBM) model of an asset price paying a continuous dividend yield of $\delta$ per annum [5] is given by the following formula

$$
d S=(r-\delta) S d t+\sigma S d z
$$

and working instead with $x=\ln S$, we obtain

$$
d x=v d t+\sigma d z, \text { where } v=r-\delta-\sigma^{2} / 2 .
$$

In a trinomial model of the asset price, over a small time interval $\Delta t$ the asset price can go up by $\Delta x$, stay the same, or go down by $\Delta x$, with probabilities $q_{u}, q_{m}$ and $q_{d}$ respectively. $\Delta x$ is called the space step of the process. This movement of the asset price is depicted in two different ways in Figure 2.1.

View (a) in Figure 2.1 is the traditional way to view the trinomial process [5]. View (b), however, is the one that we will adapt in this work as it will facilitate the exposition of the computations performed in the proposed parallel algorithm and also leads to the minimization of parallel time in the parallel algorithm. The drift and volatility parameters of the continuous time process are captured by $\Delta x, q_{u}, q_{m}, q_{d}$ with $\Delta x$ chosen so that $\Delta x=\sigma \sqrt{3 \Delta t}$ (see [5]). The discretization of the GBM requires that the drift and volatility parameters match the first two moments (mean and variance) of the distribution and the probabilities $q_{u}, q_{m}, q_{d}$ add up to one [5]. 


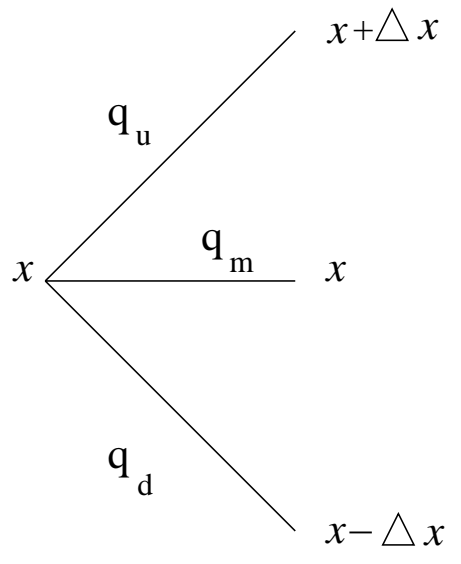

(a)

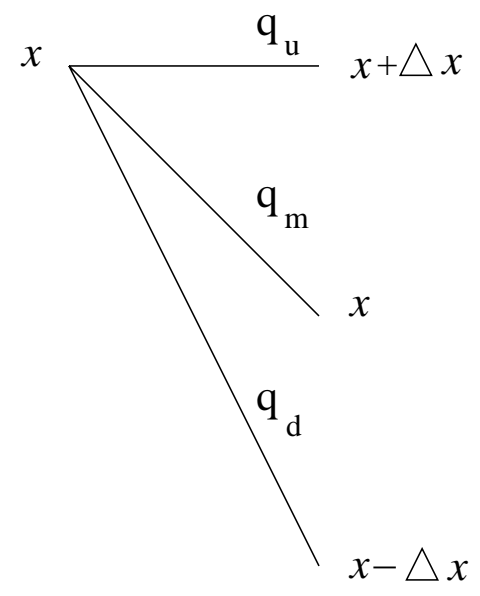

(b)

FIG. 2.1. Trinomial tree model: two views.

This way the following values for $q_{u}, q_{m}$, and $q_{d}$ can be obtained.

$$
\begin{array}{r}
q_{u}=\frac{1}{2}\left(\frac{\sigma^{2} \Delta t+v^{2} \Delta t^{2}}{\Delta x^{2}}+\frac{v \Delta t}{\Delta x}\right), q_{m}=1-\frac{\sigma^{2} \Delta t+v^{2} \Delta t^{2}}{\Delta x^{2}}, \\
q_{d}=\frac{1}{2}\left(\frac{\sigma^{2} \Delta t+v^{2} \Delta t^{2}}{\Delta x^{2}}-\frac{v \Delta t}{\Delta x}\right) .
\end{array}
$$

The trinomial process of Figure 2.1 can be extended to form a trinomial-tree such as the one depicted in Figure 2.2 in two different ways. Figure 2.2 depicts a trinomialtree for $n=3$ time steps, i.e. of horizon $n=3$. The number of levels in such a tree is $n+1=4$ and the number of nodes at any level $i$ is equal to $2 i+1$. A node in the tree is indexed by a pair $(i, j)$, where $i$ indicates the level and $j$ the distance from the top. Time $t$ can then be inferred from the level index $i: t=i \Delta t$. The standard view of a trinomial-tree is Figure 2.2 (a). In the standard view upward and downward moves point upwards and downwards to the right respectively, and steady asset moves point horizontally to the right. In this work we use the alternative view depicted in Figure 2.2(b). In the alternative view upward moves point to the right horizontally and downward moves point steeply downwards to the right, whereas steady moves point downwards to the right but at a less steeper angle. In such a view, node $(i, j)$ is thus connected to node $(i+1, j)$ (upward move), to node $(i+1, j+1)$ (steady move), and to node $(i+1, j+2)$ (downward move). Row $j$ also contains $n+1-j$ nodes in the alternate view. The option price and the asset price at node $(i, j)$ are given by $C[i, j]=C_{i, j}$ and $S[i, j]=S_{i, j}$ respectively. The latter is straightforward to compute from the number of up and down moves required to reach $(i, j)$ from $(0,0)$ and is given by the following expression for the alternative view of Figure 2.2(b).

$$
S[i, j]=S[0,0] \cdot \exp ((i-j) \cdot \Delta x) .
$$

Time step $n$ corresponds to maturity, i.e. $T=n \Delta t$. The option prices at maturity (i.e. time step $n$ ) are determined by the pay off and thus for a call option (intent to 
buy the asset at a predetermined strike price $)$ this is $C_{n, j}=\max \left(0, S_{n, j}-K\right)$ and for a put option (intent to sell) this is $C_{n, j}=\max \left(0, K-S_{n, j}\right) . K$ is the strike price at maturity $T=n \Delta t$ for a European option, and the strike price at any time before or on maturity for an American option.

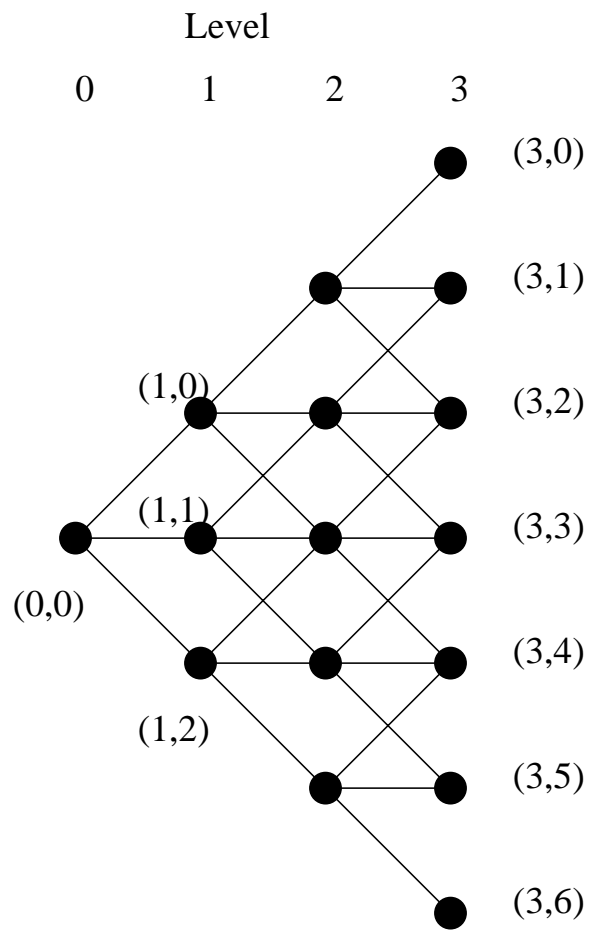

(a)

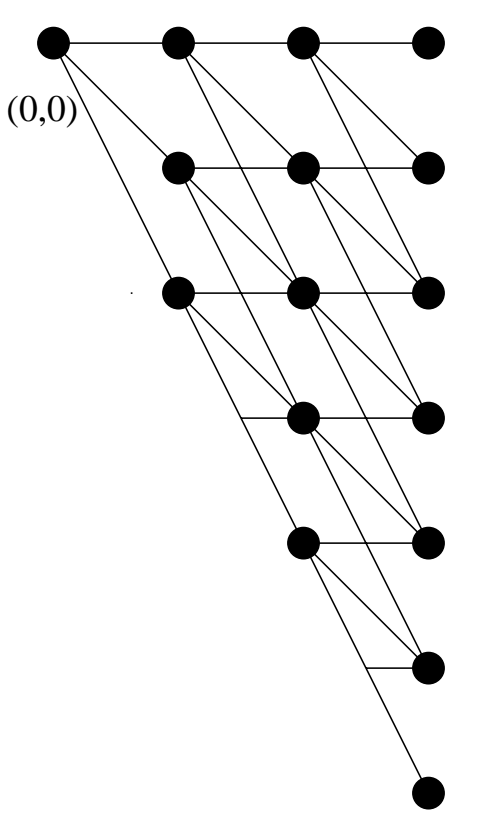

$(3,0)$

(b)

FIG. 2.2. A Trinomial Tree: two views for $n=3$

Then one can compute option prices as discounted expectations under a riskneutral assumption, and thus option prices at earlier nodes can be computed as discounted expectations. For an American put option (that can be exercised at any time before or on maturity), for $i<n$,

$$
C_{i, j}=\max \left(e^{-r \Delta t}\left(q_{u} C_{i+1, j}+q_{m} C_{i+1, j+1}+q_{d} C_{i+1, j+2}\right), K-S_{i, j}\right)
$$

(for an American call option use $S_{i, j}-K$ instead of $K-S_{i, j}$ ), whereas for a European call option that can be exercised on maturity only, for $i<n$,

$$
C_{i, j}=e^{-r \Delta t}\left(q_{u} C_{i+1, j}+q_{m} C_{i+1, j+1}+q_{d} C_{i+1, j+2}\right) .
$$

The expression for $C_{n, j}$ along with Eq. (2.4), and Eq. (2.5) or Eq. (2.6) can be used to compute the option price at every time step and node of the trinomial-tree. The option price at the start is thus $C_{0,0}$. We can extend the trinomial tree into a grid by adding nodes the way we show in Figure 2.3 so that the number of nodes in level $i$ becomes $2 n+1$. The added nodes to the original trinomial tree of Figure 2.3 are the non black-filled nodes depicted there. When we extend a trinomial tree 
Level
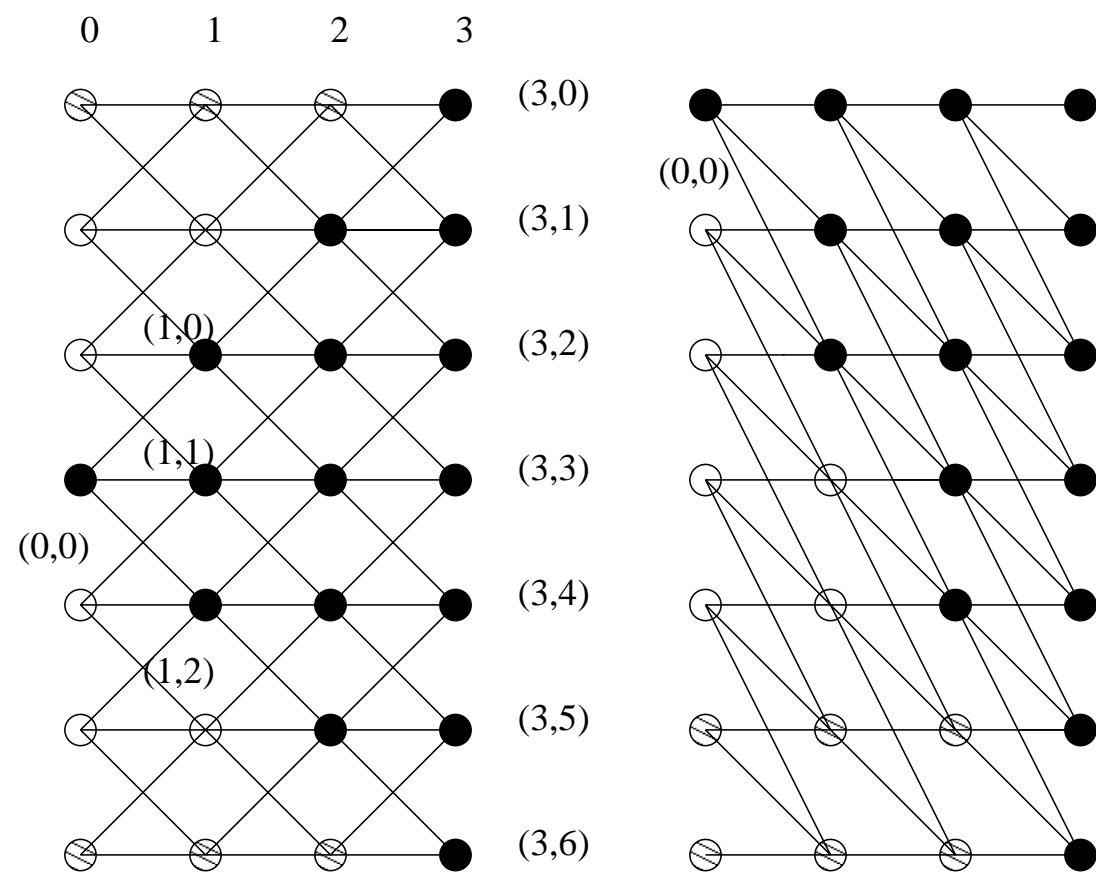

(a)

(b)

- trinomial tree nodes

$\bigcirc$ other extended grid nodes

$\vartheta$ boundary extended grid nodes

FIG. 2.3. A Trinomial Tree and its extension to a grid: two views for $n=3$

into a grid we face a boundary value problem. Consider for example Figure 2.3(a). The option prices at nodes (non black-filled) of the first row and the last row can not be computed using discounted expectations since these would require the knowledge of option prices in the rows above and beneath the specified rows that do not exist. Therefore with the currently available information only option prices at the nodes of the original trinomial tree (black-filled nodes) can be computed. Similarly, with respect to Figure 2.3(b) option prices at nodes (non black-filled) of the last two rows can not be computed either. To valuate these additional nodes of the grid we must add [5] boundary conditions such as

$$
\frac{\partial C}{\partial S}=1 \text { for } S \text { large. }
$$

$$
\frac{\partial C}{\partial S}=0 \text { for } S \text { small. }
$$

Using finite differences, Eq. (2.7) and Eq. (2.8) then yield for the extended grid of Figure 2.3(a) and for a European call option the following boundary conditions for 
the option prices of nodes of rows 0 and $2 n$.

$$
\begin{gathered}
\frac{C_{i, 0}-C_{i, 1}}{S_{i, 0}-S_{i, 1}}=1 \Rightarrow C_{i, 0}=C_{i, 1}+S_{i, 0}-S_{i, 1} \\
\frac{C_{i, 2 n-1}-C_{i, 2 n}}{S_{i, 2 n-1}-S_{i, 2 n}}=0 \Rightarrow C_{i, 2 n}=C_{i, 2 n-1} .
\end{gathered}
$$

For the alternate view of Figure 2.3 (b) option prices of the last two rows cannot be determined; even though Eq. (2.7) does not apply, Eq. (2.8) yields boundary conditions for these nodes and option prices that are given by the following expressions.

$$
\begin{aligned}
\frac{C_{i, 2 n-1}-C_{i, 2 n}}{S_{i, 2 n-1}-S_{i, 2 n}} & =0, \quad \frac{C_{i, 2 n-2}-C_{i, 2 n-1}}{S_{i, 2 n-2}-S_{i, 2 n-1}}=0 \Rightarrow \\
C_{i, 2 n-1} & =C_{i, 2 n-2}, \quad C_{i, 2 n}=C_{i, 2 n-1} .
\end{aligned}
$$

Similarly, for an American-style put option the corresponding boundary conditions are as follows.

$$
\begin{aligned}
& \frac{C_{i, 2 n-1}-C_{i, 2 n}}{S_{i, 2 n-1}-S_{i, 2 n}}=1, \frac{C_{i, 2 n-2}-C_{i, 2 n-1}}{S_{i, 2 n-2}-S_{i, 2 n-1}}=1 \Rightarrow \\
& (2.12) \quad C_{i, 2 n-1}=C_{i, 2 n-2}+S_{i, 2 n-1}-S_{i, 2 n-2} \quad, \quad C_{i, 2 n}=C_{i, 2 n-1}+S_{i, 2 n}-S_{i, 2 n-1} .
\end{aligned}
$$

We now show how the explicit finite difference method can be applied to the BlackScholes partial differential equation. Consider the Black-Scholes [3] partial differential equation for valuating the option price $C$ of an asset $S$ that follows GBM given by Eq. (2.1) before and after the substitution $x=\ln S$.

$$
\begin{aligned}
-\frac{\partial C}{\partial t} & =\frac{1}{2} S^{2} \sigma^{2} \frac{\partial^{2} C}{\partial S^{2}}+(r-\delta) S \frac{\partial C}{\partial S}-r C \text { or } \\
-\frac{\partial C}{\partial t} & =\frac{1}{2} \sigma^{2} \frac{\partial^{2} C}{\partial x^{2}}+v \frac{\partial C}{\partial x}-r C .
\end{aligned}
$$

By replacing partial derivatives with forward differences for $\partial C / \partial t$ and central differences for $\partial^{2} C / \partial x^{2}$ and $\partial C / \partial x$ we obtain for the view of Figure 2.3(a) the following.

$$
\begin{array}{r}
-\frac{C_{i+1, j}-C_{i, j}}{\Delta t}=\frac{1}{2} \sigma^{2} \frac{C_{i+1, j-1}-2 C_{i+1, j}+C_{i+1, j+1}}{\Delta x^{2}}+ \\
v \frac{C_{i+1, j-1}-C_{i+1, j+1}}{2 \Delta x}-r C_{i+1, j}
\end{array}
$$

or equivalently,

$$
C_{i, j}=p_{u} C_{i+1, j-1}+p_{m} C_{i+1, j}+p_{d} C_{i+1, j+1},
$$


where

$$
\begin{array}{r}
p_{u}=\frac{1}{2}\left(\frac{\sigma^{2} \Delta t}{\Delta x^{2}}+\frac{v \Delta t}{\Delta x}\right), p_{m}=1-\frac{\sigma^{2} \Delta t}{\Delta x^{2}}-r \Delta t, \\
p_{d}=\frac{1}{2}\left(\frac{\sigma^{2} \Delta t}{\Delta x^{2}}-\frac{v \Delta t}{\Delta x}\right) .
\end{array}
$$

Eq. (2.15) is equivalent to taking discounted expectations. If, however, in Eq. (2.14) the last term $-r C_{i+1, j}$ becomes $-r C_{i, j}$, then Eq. (2.14) is written in the form of Eq. (2.17).

$$
\begin{array}{r}
-\frac{C_{i+1, j}-C_{i, j}}{\Delta t}=\frac{1}{2} \sigma^{2} \frac{C_{i+1, j-1}-2 C_{i+1, j}+C_{i+1, j+1}}{\Delta x^{2}}+ \\
v \frac{C_{i+1, j-1}-C_{i+1, j+1}}{2 \Delta x}-r C_{i, j}
\end{array}
$$

and equivalently,

$$
C_{i, j}=\frac{1}{1+r \Delta t}\left(p_{u} C_{i+1, j-1}+p_{m} C_{i+1, j}+p_{d} C_{i+1, j+1}\right),
$$

where

$$
p_{u}=\frac{1}{2}\left(\frac{\sigma^{2} \Delta t}{\Delta x^{2}}+\frac{v \Delta t}{\Delta x}\right), p_{m}=1-\frac{\sigma^{2} \Delta t}{\Delta x^{2}}, p_{d}=\frac{1}{2}\left(\frac{\sigma^{2} \Delta t}{\Delta x^{2}}-\frac{v \Delta t}{\Delta x}\right),
$$

and $1 /(1+r \Delta t)$ approximates $1 / \exp (r \Delta t)$. Therefore the explicit finite difference method for the solution of the Black-Scholes equation given by Eq. (2.14) and Eq. (2.15) is equivalent to a discrete trinomial process [5] as given by Eq. (2.17) and Eq. (2.18).

Returning to the Black-Scholes PDE, Eq. (2.15) can be written as follows with reference to the grid of Figure 2.3 (b)

$$
C_{i, j}=\left(p_{u} C_{i+1, j}+p_{m} C_{i+1, j+1}+p_{d} C_{i+1, j+2}\right) .
$$

The program of Table 2.1 provides pseudocode for the valuation of an American put option using the explicit finite difference method and Eq. (2.20).

3. A parallelization of the EFDM computations. The extended grid used for the solution of the Black-Scholes PDE with the EFDM has $(n+1)(2 n+1)$ nodes, as the grid has $n+1$ levels and $2 n+1$ rows. Depending on the application, the option price at node $(0,0)$ may only be required. The pseudocode in Table $(2.1)$ reflects this. Information related to option prices is maintained in array $C$ and an auxiliary array is also used in the valuations in the form of array $C^{*}$; asset prices are maintained in array $S$. Total space requirements are thus $3(2 n+1)$, where $n$ is the time horizon. In some other cases option prices on all nodes need to become available at the conclusion of the valuation. Our algorithm extends easily to this latter case by increasing memory requirements to $(n+1)(2 n+1)+2(2 n+1)$ from $3(2 n+1)$ as the one-dimensional $C$ becomes a two-dimensional array.

We give an outline of the proposed parallel algorithm BSP_EFDM by describing how data are distributed and how computation and communication is scheduled. Let 


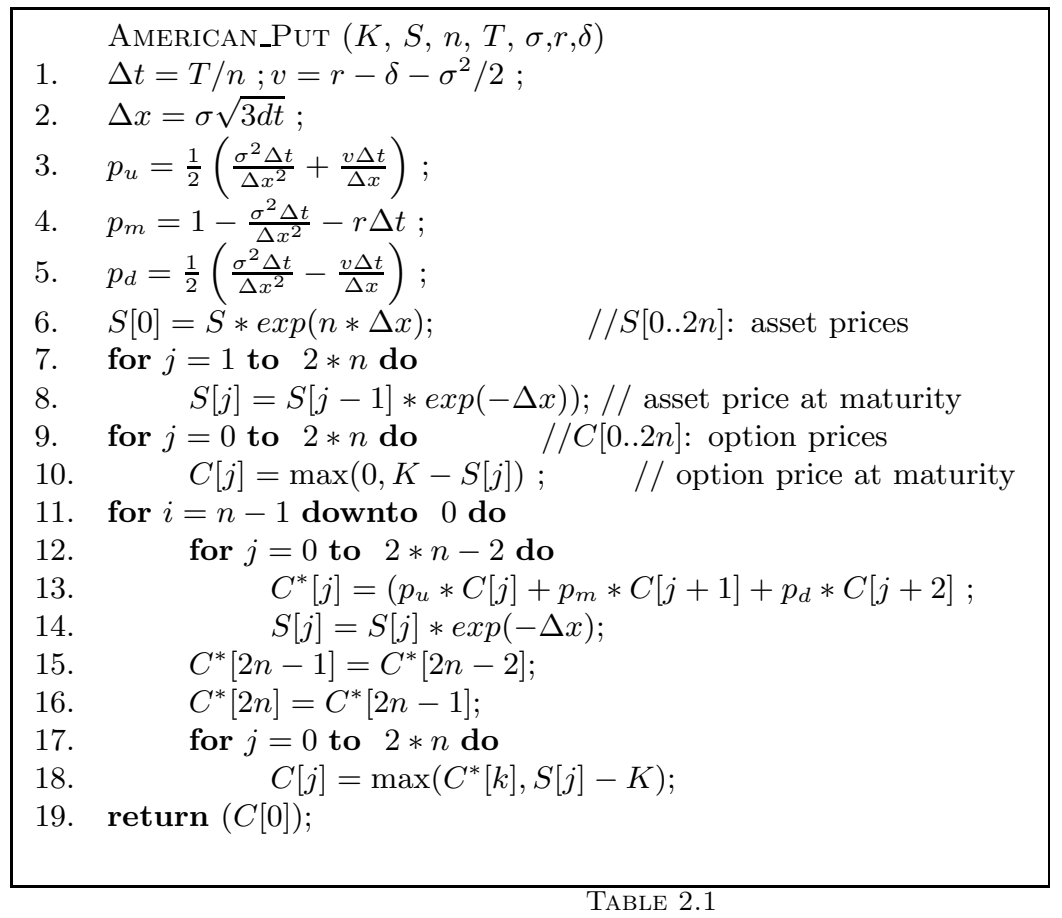

Procedure AMERICAN_Put for the explicit finite difference method.

\section{BSP_EFDM ()}

// Note: We use a two dimensional array $C_{i, j}$ to refer to the

// option price of the node in row $j$ of level $i$

1. Assign $C_{n, j}$ prices to Block 0 nodes (i.e. initialize level $n$ );

2. for $(k=n ; k>0 ; k=k-b+1)\{$

3. Block 1 nodes are valuated (lines 11-14 and 17-18 are executed);

4. Block 2 node information is sent from processor $m$ to processor $m-1$;

5. $\quad$ Block 3 nodes are valuated (lines 11-14 and 17-18 are executed);

In addition, processor $p-1$ executes lines 15-16 to establish option prices for its last two rows.

\} //Option prices for levels $k-b+1, \ldots, k$ have been valuated.

TABLE 3.1

Procedure BSP_EFDM.

$n \geq p$. We distribute the three arrays $C, C^{*}, S$ evenly among the $p$ processors in a contiguous column blocked distribution so that each processor gets $\lfloor(2 n+1) / p\rfloor$ or $\lceil(2 n+1) / p\rceil$ nodes. Given this partitioning scheme the maximum imbalance between any two processors is 1 .

We will also introduce a parameter $b$ that will be called the blocking factor of the parallel execution of EFDM, and we require that $\lfloor(2 n+1) / p\rfloor>2 b$. The value of $b$ determines the optimality of the parallel algorithm and its latency tolerance, and itself can be determined from the characteristics of the underlying parallel system as expressed in terms of the BSP parameters $p, L$, and $g$. Parameter $b$ will be kept small 
$b=o(n / p)$, although $b$ is chosen so that $b=\omega(1)$.

Parallel algorithm BSP_EFDM processes $b$ levels of the $(2 n+1) \times(n+1)$ grid at a time in a single update starting from the rightmost level $n$ and working towards level 0 . This is done to avoid frequent communication and synchronization operations. In fact the algorithm performs two supersteps for every $b$ levels of the grid: a superstep that includes both computation and communication and a computation only superstep. The memory allocation scheme that subdivides the grid into rectangles allows each processor to perform the relevant update operations blockwise. Figure 3.1 depicts part of the grid for two neighboring processors and $b$ consecutive levels and highlights how the parallel valuation of the nodes is scheduled to achieve optimality. The nodes whose valuation will be described are black-filled and belong to some processor, and let us call it processor zero. Node dependencies of the black-filled nodes are shown in Figure 3.1; only a fraction, however, of the black-filled nodes are shown (the bottom $2 b$ rows). The white-filed nodes belong to a neighboring processor and let processor one be the processor after processor zero. Nodes of levels before and after the depicted levels and on rows before or after the depicted rows are not shown, nor are the full dependencies of the empty-filled nodes shown.

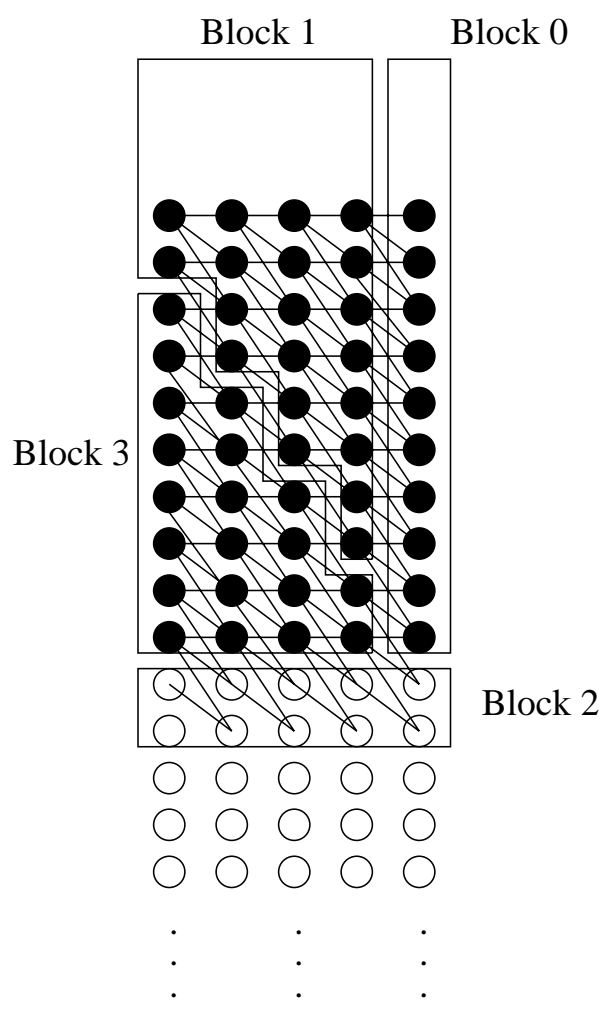

FIG. 3.1. Parallel valuation scheduling using EFDM $(b=5)$

For the sake of clarity let us assume that $p$ divides $2 n+1$ so that each processor gets $(2 n+1) / p$ consecutive nodes of a level. At some step of BSP_EFDM we are 
interested in valuating the nodes of some processor, say processor zero holding a block of $(2 n+1) / p \times b$ nodes. These are the black-filled nodes partially depicted in Figure 3.1. We call this set of nodes Block A. This Block A of nodes is further subdivided into three blocks of nodes: Block 0,1 and 3. Block 0 is an $(2 n+1) / p \times 1$ array; column $i$ (from right) of Block 1 is an $((2 n+1) / p-2 i) \times 1$ array, where $i=1, \ldots, b-1$. Column $i$ (from right) of Block 3 is a $2 i$ array, where $i=1, \ldots, b-1$. Nodes in Block 1 and Block 3 are valuated following the sequential algorithm of Table 2.1, i.e. right to left and top to bottom. An invariant that will be maintained in BSP_EFDM is that Block 0 nodes have already been valuated, when the Block A nodes are to be valuated. This invariant is easy to maintain because in the boundary (initial) case, Block 0 nodes consist of nodes of level $n$ and these nodes are valuated separately in lines 6-10 in Table 2.1 before the main loop of lines 11-18 that valuates the remaining nodes; this preprocessing step is retained in the parallel algorithm.

Block 1 nodes can be valuated using locally to processor 0 (i.e. Block A) available information because such nodes only need the option prices of Block 0 nodes and the sequential schedule of the program of Table 2.1 to complete the valuation. Block 3 nodes stored in processor zero, however, require non-local information that is available to processor one (the next processor); this required information is available to the Block 2 white-filled nodes that reside in processor one. Therefore Block 2 option prices need to be communicated from processor one to processor zero as soon as they become available i.e. as soon as the corresponding nodes of processor one are valuated. The nodes forming Block 2 are themselves Block 1 and Block 0 nodes to processor one. This is because Block 3 extends to $2(b-1)$ rows from the bottom of Block A and Block 2 nodes belong to the two top rows of a processor block. As long as $\lfloor(2 n+1) / p\rfloor>2 b$, the Block 2 nodes are Block 1 nodes for processor one. Therefore option prices for Block 2 nodes can be communicated as soon as Block 1 and Block 0 nodes have been valuated in parallel in all participating processors. Processor $p-1$ in particular does not need to wait for Block 2 option prices. It computes the option prices of its last two rows using the boundary conditions of lines 15-16 of the program of Table 2.1 derived from equation 2.12.

The work schedule performed by an arbitrary processor $m-1$ for any Block A valuations is listed below noting that the invariant related to Block 0 nodes is maintained.

o. Block 0 nodes in processor $m-1$ have already been valuated.

i. Block 1 nodes in processor $m-1$ and in parallel with other processors are valuated using the option prices of Block 0 nodes.

ii. Block 2 nodes are Block 0 and Block 1 nodes in processor $m$ provided that $\lfloor(2 n+1) / p\rfloor \geq 2 b$ and have thus already been valuated. Option prices of Block 2 nodes are communicated to processor $m-1$ from processor $m$.

iii. Block 3 nodes in processor $m-1$ and in parallel with other processors are then valuated.

Steps (i)-(iii) are repeated until the nodes of all levels of the grid are valuated. Steps (i) and (ii) form a superstep and step (iii) forms another superstep.

3.1. Algorithm BSP_EFDM. The pseudocode in Table 3.1 describes the major steps of an EFDM based parallel option price valuation. All references to lines of code are to the code of AMERICAN_PuT depicted in Table 2.1. With reference to Table 3.1 for algorithm BSP_EFDM, we analyze the time requirements of the parallelization of the valuation method described in Table 2.1. Note that $C$ is depicted as a twodimensional array in Table 3.1 to facilitate exposition only. 
Step 1. The $2 n+1$ nodes of the grid at time $n$ are split into groups and each processor gets $\lfloor(2 n+1) / p\rfloor$ or $\lceil(2 n+1) / p\rceil$ nodes The initialization of step 1 of Table 3.1 thus requires time proportional to $\lceil(2 n+1) / p\rceil \leq(2 n+1) / p+1$.

Loop of Steps 2-5. The loop of step 2 starts at $k=n$, and every time it is executed, all the nodes of the grid of levels $k-b+1 \ldots k-1$ are valuated; we call the valuation of all the nodes of the grid in a single iteration of step 2, an update. For any value of $k$, the $i$-th update, where $i=\lfloor(n-k+(b-1)) /(b-1)\rfloor$, thus involves levels $k-b+1 \ldots k-1$. Eventually $k$ reaches a non-positive value and the loop terminates. The total number of updates performed until $k$ reaches 0 or the number of times the loop of step 2 is executed is $\lceil n /(b-1)\rceil$. In calculations later in this section, for simplicity and without loss of generality, we will assume that $n$ is a multiple of $b-1$.

Steps 3 and 5. Each processor is assigned a block of approximately the same dimensions $\lfloor(2 n+1) / p\rfloor \times b$ or $\lceil(2 n+1) / p\rceil \times b$. The total work required for the $i$-th update is thus equal to $(2 n+1) b / p+b$ per processor. However this work is split into two supersteps. In step 3, each processor valuates the nodes of Block 1, i.e. it performs at most

$$
\sum_{i=1}^{b-1}((2 n+1) / p+1-2 * i)=(2 n+1)(b-1) / p-(b-1)^{2}
$$

node valuations. In step 5 each processor valuates at most

$$
\sum_{i=1}^{b-1} 2 * i=b(b-1)
$$

nodes of Block 3. In the remainder we are going to make the assumption that $n$ is a multiple of $b-1$, an assumption that does not affect the asymptotics of the calculations below.

Step 4. The communication performed in step 4 involves the communication of $2 b$ option prices, the size of Block 2, per processor.

Let $N=\left\lceil\frac{n}{(b-1)}\right\rceil$. The parallel time of steps 3 through 5 is thus given by the following expression.

$$
\begin{array}{r}
\sum_{i=1}^{N}\left(\max \left\{L,(2 n+1)(b-1) / p-(b-1)^{2}+g 2 b\right\}+\max \{L, b(b-1)\}\right) \leq \\
\sum_{i=1}^{N}\left(2 L+2 g b+\frac{(2 n+1)(b-1)}{p}+(b-1)\right)= \\
N\left(2 L+2 g b+\frac{(2 n+1)(b-1)}{p}+(b-1)\right)= \\
\frac{2 L n}{b-1}+\frac{(2 n+1) n}{p}+n+\frac{2 g n b}{b-1} .
\end{array}
$$

The total parallel time results by combining the time for step 1 with that for steps 3,4 , and 7 .

$$
\begin{aligned}
T(n, p) & =\frac{2 n+1}{p}+1+\frac{2 L n}{b-1}+\frac{(2 n+1) n}{p}+n+\frac{2 g n b}{b-1} \\
& =\frac{2 L n}{b-1}+\frac{(2 n+1)(n+1)}{p}+n+1+\frac{2 g n b}{b-1} .
\end{aligned}
$$


We note that the sequential computation time is given by $T(n)=\sum_{i=0}^{n}(2 n+1)=$ $(2 n+1)(n+1)$. The following Theorem can then be derived.

THEOREM 3.1. For any integer $n, p \leq n, b \geq 2$ such that $b-1$ divides $n$, and $\lfloor(2 n+1) / p\rfloor \geq 2 b$, algorithm BSP_EFDM for option-price valuations using the explicit finite difference method requires parallel computation time equal to

$$
C(n, p)=\frac{(2 n+1)(n+1)}{p}+n+1
$$

and parallel communication (and synchronization) time equal to

$$
M(n, p, L, g)=\frac{2 L n}{b-1}+\frac{2 g n b}{b-1}
$$

One-optimality for BSP_EFDM can be achieved for a wide range of the BSP parameters $L$ and $g$. The following corollary describes such a case.

Corollary 3.2. For any integer $n, p \leq n, b \geq 2$ such that $b-1$ divides $n$, and $\lfloor(2 n+1) / p\rfloor \geq 2 b$, algorithm BSP_EFDM for $L=o(n b / p)$, and $g=o(n / p)$ achieves $\pi=1+o(1)$ and $\mu=o(1)$.

Proof. For $\operatorname{Ln} /(b-1)=o\left(n^{2} / p\right)$ synchronization periodicity costs are absorbed in the low order terms of the running time. Therefore for $L=o(n b / p)$, such costs become insignificant. In computation time, the overhead of the blocked allocation of nodes to processors is reflected in the contributing term $(n+1)$. The contributions of communication time become negligible as long as $g=o(n / p)$. Note that blocking affects only synchronization time; the larger the $b$ the fewer synchronizations need to be performed or the larger $L$ can be, i.e. the algorithm increases its latency tolerance. By dividing either $C(n, p)$ or $M(n, p, L, g)$ by sequential running time $T(n)$ we deduce that for the given conditions of $L, b$ and $g$ we have that $\pi=1+O(p / n)$ and $\mu=o(1)$. The corollary then follows.

4. Experimental Results. A parallel program designed under the BSP programming paradigm and thus viewed as a sequence of supersteps, with the required communication occurring at the end of each superstep can also be realized as a library of functions with architecture independent semantics for process creation, remote data access and bulk synchronization. The Oxford BSP Toolset, BSPlib, implements such a paradigm [14] and provides specific library support for BSP programming. The library functions of the Oxford BSP Toolset are callable from standard imperative languages such as $\mathrm{C}$ and Fortran. The effort required to learn the basics of BSPlib is minimal as it consists of about 10-15 functions. BSP-model oriented programming support does not require the use of BSP-specific libraries such as BSPlib; non BSPspecific libraries such as MPI (Message Passing Interface) that also support the simple communication and synchronization primitives required for programming under the BSP model can also be used to implement algorithms and programs written under the BSP programming framework. LAM-MPI [15] and Open MPI [11] are two such examples. Even SWARM [1], a parallel computing framework for multi-core processors, allows, though with some modifications, for a BSP style of programming.

We have implemented latency tolerant algorithm BSP_EFDM under the BSP framework in a transparent and portable way. We have thus made our BSP code that implements BSP_EFDM run transparently not only on a BSP-specific library, BSPlib, but also on MPI, a non BSP-specific framework, through the LAM-MPI and Open MPI implementations of MPI. We have written our code in an architecture and 
also library independent way using standard ANSI C and few generic communication primitives/functions related to processor creation, destruction, identification, and direct memory interprocessor communication. These generic communication primitives are then translated at compile-time into specific function calls of BSPlib or LAMMPI/Open MPI. For the sake of an example, if BSPlib is used, direct memory communication is performed through the bsp_put, bsp_get and similar calls of BSPlib. If MPI is chosen instead, direct memory communication is performed through the corresponding operations MPI_Put, MPI_Get that are part of the MPI-2 extensions that are supported by LAM-MPI. No change in the source code is required to make the program run under one or the other library. Therefore we can claim that our code is not only architecture independent but also parallel programming/communication library independent. Since SWARM provides very limited interprocessor communication support, our code [9] is not as flexible in working with SWARM in a multi-core environment. However, both BSPlib and Open MPI, at first glance, seem to be quite robust under a multi-core environment.

The implementation language is ANSI $\mathrm{C}$ and the code was tested for scalability on a cluster of 16 dual-processor PC workstations with PentiumII 350Mhz processors (128MB RAM total), Redhat Linux 7.1-equipped with communication performed through 100Mbit 3Com-905B Ethernet cards and the workstations connected by a 24port CISCO Catalyst 2924M-XL-EN switch. The default GNU Project's gcc compiler is used through either the BSPlib or the LAM-MPI front-end, and the source-code is compiled with the -03 compiler option set. Timing is obtained through the use of the real-time (wall-clock time) clock functions available in both libraries which are (bsp_time of BSPlib [10] and MPI_Wtime of MPI/LAM-MPI). All results and variables used for communication and computation involve ANSI C double 64-bit data types, later referred to as word of information. In addition we tested how suitable the implementation of BSP_EFDM is in a limited multi-core environment; no scalability study took place. For these dual-core experiments, an Intel dual-core T5600 $1.83 \mathrm{Ghz}$ processor was used in a default Fedora Core 7 installation. The same BSPlib version was used except that multi-core communication and synchronization support were provided through SHMEM_SYSV. For Open MPI the default configuration for version 1.2.7 was used. For SWARM, version 1.1 at [2] was used. The same default gcc compilation options were used for all three libraries that were used in the other PC cluster experiments. The code developed for this experimental study is publically available at the author's Web-page [9].

The tested platform is in an non-SMP configuration the 16-node cluster utilizing only one CPU per node, and in an SMP configuration the 16-node cluster utilizing both CPUs for a total of 32 active CPUs. When experimental results are reported for say a four-processor subcluster in the former configuration all four CPUs belong to different nodes whereas in the latter configuration they belong to only two nodes utilizing both CPUs of each node. The reported speedup results reflect valuations of American-style put options; a report on the performance and relative merits of the implementation of BSP_EFDM is also included. Timing results are reported for $p=1$ which are independent of $b$ and speedup results are reported for other processor configurations with figures truncated to two decimal digits. Such results are averages of four experiments. We used a sequential implementation to derive these timing figures for $p=1$; we did not use the parallel implementation run on a single processor. For processor sizes $p=4,8,16,32$, relative speedup figures are only reported rather than actual timing results; it is straightforward to obtain time 
from these speedup figures and the $p=1$ timing results, if necessary. We performed experiments for problem sizes $n=8192,16384,32768$. We also varied block size $b$; in the experiments we set $b=65,129,257,513$, and 1025 . Note that our code has been made to work for any value of $b>1$ and does not require that $b-1$ divide $n$.

TABLE 4.1

Speedup results using BSPlib on an non-SMP PC-cluster.

\begin{tabular}{|c|c|c|c|c|c|c|c|c|c|}
\hline & \multicolumn{3}{|c|}{$n=8192$} & \multicolumn{3}{|c|}{$n=16384$} & \multicolumn{3}{|c|}{$n=32768$} \\
\hline & \multicolumn{3}{|c|}{$p=1: T=21.93$} & \multicolumn{3}{|c|}{$p=1: T=130.44$} & \multicolumn{3}{|c|}{$p=1: T=589.61$} \\
\hline & \multicolumn{3}{|c|}{ Speedup } & \multicolumn{3}{|c|}{ Speedup } & \multicolumn{3}{|c|}{ Speedup } \\
\hline$b$ & $p=4$ & $p=8$ & $p=16$ & $p=4$ & $p=8$ & $p=16$ & $p=4$ & $p=8$ & $p=16$ \\
\hline 65 & 4.36 & 7.41 & 11.25 & 6.57 & 12.57 & 19.50 & 5.62 & 13.54 & 25.33 \\
\hline 129 & 4.50 & 8.15 & 13.05 & 6.60 & 12.93 & 21.49 & 5.94 & 13.71 & 26.75 \\
\hline 257 & 4.57 & 8.50 & 14.06 & 6.99 & 13.52 & 22.34 & 5.98 & 13.87 & 27.45 \\
\hline 513 & 4.58 & 8.60 & 14.43 & 6.91 & 13.46 & 22.92 & 6.23 & 14.61 & 27.82 \\
\hline 1025 & 4.50 & 8.34 & - & 6.68 & 13.35 & 22.65 & 6.05 & 15.26 & 27.72 \\
\hline
\end{tabular}

Table 4.1 shows timing/speedup results for problem sizes $n=8192,16384,32768$ on the 16-node PC-cluster (one CPU per node). Table 4.2 shows corresponding results for the same problem sizes on an SMP configuration of the cluster. A surprising observation is the effect of caching in almost all cases that leads to superlinear speedup figures despite the high-latency of the PC cluster. Only for $n=8192$ and $p=16$ and in one case for $(n, p, b)=(8192,8,65)$ did we observe speedup figures less than $p$. The reported block sizes are the ones that provide a minimization of parallel running time (and thus maximization of speedup). Block sizes $b>1025$ and $b<65$ do not improve performance. For problem size $n=8192, b$ is limited according to Theorem 3.1 to values up to $b=513$ for $p=16$. Speedup figures for large problem sizes reach as high as $150 \%$ of $p$. An interesting observation is that the figures of Table 4.2 are worse/slightly worse than those of Table 4.1 for $p=4,8$ and similar to slightly better for $p=16$, a possible result of caching effects.

Two conclusions can be immediately drawn from these tables: the scalability of BSP_EFDM and its tolerance to high latency in communication. The introduction of the block factor $b$ in BSP_EFDM contributes to this.

Table 4.1 indicates that the best performance for $n=8192$ is achieved for $b$ around $b=513$, for $n=16384$ around $b=257-513$ and for $n=32768$ around $b=513-1025$. Similar ranges were observed in the reported results of Table 4.2. Some fluctuations observed especially for problem sizes $n=32768$ are due either to the non divisibility of $n$ by $b$ or external factors (e.g. other programs running in the system).

Overall, three factors affect performance: communication, the block factor $b$ that increases processor utilization within a superstep and latency issues related to bulksynchronization operations.

From the expression for communication time in Theorem 3.1 the contributions of communication are minimized provided $2 g n b /(b-1)$ is kept smaller than $(2 n+$ 1) $(n+1) / p$ or in other words the ratio $R_{1}=p g / n$ is minimized. Similarly, the ratio $2 L n /(b-1)$ over $(2 n+1)(n+1) / p$ must also be minimized so that $R_{2}=L g / b n$ is also kept small. Thefore a lower bound on the attainable speedup is $S=p /\left(1+R_{1}+R_{2}\right)$. 
TABLE 4.2

Speedup results using BSPlib on an SMP PC-cluster.

\begin{tabular}{rrrrrrrrrr}
\hline \multicolumn{4}{c}{$n=8192$} & \multicolumn{3}{c}{$n=16384$} & \multicolumn{3}{c}{$n=32768$} \\
\hline \multicolumn{4}{c}{$p=1: T=21.93$} & \multicolumn{3}{c}{$p=1: T=130.44$} & \multicolumn{3}{c}{$p=1: T=589.61$} \\
\hline \multicolumn{4}{c}{ Speedup } & \multicolumn{3}{c}{ Speedup } & \multicolumn{3}{c}{ Speedup } \\
\hline$b$ & $p=4$ & $p=8$ & $p=16$ & $p=4$ & $p=8$ & $p=16$ & $p=4$ & $p=8$ & $p=16$ \\
\hline 65 & 4.32 & 7.12 & 10.70 & 6.61 & 11.90 & 19.10 & 5.25 & 13.98 & 25.37 \\
\hline 129 & 4.47 & 7.75 & 12.39 & 6.67 & 12.62 & 21.42 & 5.26 & 14.15 & 25.54 \\
\hline 257 & 4.55 & 8.15 & 14.06 & 6.80 & 12.89 & 22.57 & 5.33 & 14.35 & 27.71 \\
\hline 513 & 4.58 & 8.34 & 14.43 & 6.85 & 13.02 & 23.13 & 5.60 & 14.55 & 28.18 \\
\hline 1025 & 4.49 & 8.09 & - & 6.63 & 12.91 & 23.09 & 5.31 & 14.05 & 28.05 \\
\hline
\end{tabular}

TABLE 4.3

$L$ and $g$ parameters of the cluster for varying library and processor configurations.

\begin{tabular}{rcc}
\hline $\begin{array}{c}\text { Library/Cluster } \\
\text { configuration }\end{array}$ & $L$ & $g=16$ \\
\hline BSPlib non-SMP & $4000 \mu$ secs & $6 \mu$ secs $/$ word \\
\hline BSPlib SMP & $5200 \mu$ secs & $12 \mu$ secs $/$ word \\
\hline LAM-MPI non-SMP & $10000 \mu$ secs & $28 \mu$ secs $/$ word \\
\hline LAM-MPI SMP & $16000 \mu$ secs & $46 \mu$ secs $/$ word \\
\hline
\end{tabular}

The effective operation rate of the CPUs of the cluster for the problem in hand varies with problem and processor size and is around 6.5 million node updates per second. We call this the "flop" rate of the application. We measured the effective BSP parameters $p, L, g$ of the cluster under both BSPlib and LAM-MPI. For $p=16$ for example, $L \approx 4000 \mu$ secs for the non-SMP configuration and $L \approx 5200 \mu$ secs for the SMP configuration. For LAM-MPI the cluster behaves as a BSP machine with $L \approx 10000 \mu$ secs and $L \approx 16000 \mu$ secs respectively. All values for $L$ and $g$ are reported in Table 4.3 for the two libraries and the two cluster configurations. These figures are results of separate experiments run on the cluster. Note that the reported figures for $g$ are much higher than the asymptotic ones observed for large communication sizes where $g$ ranges between 1.5 (non-SMP) and 1.9 (SMP) $\mu$ secs per word for BSPlib and 2.4 (non-SMP) and 1.9 (SMP) $\mu$ secs per word for LAM-MPI. The reported $\mu$ second figures are translatable into operations by multiplying them with the effective megaflop rate of the application. For BSPLib thus the figures for $L$ and $g$ are $L \approx 26000$ and $L \approx 34000$ operations respectively and $g=39$ and $g=78$ operations per word (one word is a $\mathrm{C}$ double data type). For LAM-MPI the figures for $L$ and $g$ are $L=65000$ and $L=104000$ operations respectively and $g=182$ and $g=299$ operations per word respectively for the two configurations.

Consider the case of the non-SMP configuration for $p=16$ and $n=8192$. The ratio $R_{2}=L p / b n$ has a nominator that is $L p$. This is about 420000 for the parameters $L, p$ of that configuration. In order to make the ratio $R_{2}$ less than one, since $n=8192$ is the smallest problem size, we need to have $b>64$ or about so. This is how we have decided on the range of parameter $b$. For smaller values of $b$ performance deteriorates. 
For example for BSPlib in the non-SMP configuration and $p=16, n=8192$, for block sizes $b=5,9,17,33$ the observed speedups were $2.5,4.1,6.2$, and 7.6 respectively much worse that the observed speedups of Table 4.1 that are in the range $11-14$. This signifies the importance of $b$ in making the parallel algorithm latency tolerant.

For BSPlib, $p=16, n=8192$ and the non-SMP configuration, if we calculate $R_{1}=p g / n$, then $R_{2}=L p / b n$ and finally $S=p /\left(1+R_{1}+R_{2}\right)$ we conclude that for $b=65,129,257,513$ a lower bound on the attainable speedup is 8.6, 10.9, 12.6, 13.7 . The observed speedup figures were indeed higher than these lower bounds, namely, $11.25,13.05,14.06,14.43$ respectively. Note that the effect of communication $R_{1}$ becomes significant for large values of $b$ (greater than $b=257$ ) when the contributions of $R_{2}$ become insignificant.

We have also compiled the implementation of BSP_EFDM and run similar experiments using LAM-MPI as the parallel communication library. The timing results obtained were similar to those obtained through BSPlib although slightly worse in almost all cases. The obtained results, however, provide similar and consistent high performance figures. The speedup results are reported in Table 4.4 and Table 4.5 respectively. The consistency in performance of the parallel code under two diverse parallel programming libraries seems to support our argument that parallel code can be made not only architecture independent but also communication library independent if designed under the framework of the BSP model. For $p=16, n=8192$ if we repeat the calculations of $R_{1}, R_{2}, S$ we obtain that for $b=65,129,257,513$ a lower bound on the attainable speedup is $4.7,6.8,8.6$, and 9.9. The observed values were $4.70,7.29,9.70$ and 11.79 respectively, matching or slightly exceeding the theoretical values.

TABLE 4.4

Speedup results using LAM-MPI on an non-SMP PC-cluster.

\begin{tabular}{|c|c|c|c|c|c|c|c|c|c|}
\hline & \multicolumn{3}{|c|}{$n=8192$} & \multicolumn{3}{|c|}{$n=16384$} & \multicolumn{3}{|c|}{$n=32768$} \\
\hline & \multicolumn{3}{|c|}{$p=1: T=21.93$} & \multicolumn{3}{|c|}{$p=1: T=130.44$} & \multicolumn{3}{|c|}{$p=1: T=589.61$} \\
\hline & \multicolumn{3}{|c|}{ Speedup } & \multicolumn{3}{|c|}{ Speedup } & \multicolumn{3}{|c|}{ Speedup } \\
\hline$b$ & $p=4$ & $p=8$ & $p=16$ & $p=4$ & $p=8$ & $p=16$ & $p=4$ & $p=8$ & $p=16$ \\
\hline 65 & 4.15 & 5.68 & 4.70 & 5.93 & 10.58 & 10.78 & 5.68 & 13.04 & 17.23 \\
\hline 129 & 4.39 & 6.90 & 7.29 & 6.15 & 11.93 & 14.86 & 5.74 & 13.35 & 20.90 \\
\hline 257 & 4.54 & 7.72 & 9.70 & 6.74 & 12.69 & 18.19 & 5.81 & 14.38 & 24.40 \\
\hline 513 & 4.59 & 8.18 & 11.79 & 7.08 & 13.20 & 20.57 & 5.86 & 14.54 & 26.20 \\
\hline 1025 & 4.38 & 8.15 & - & 6.53 & 13.15 & 20.80 & 6.12 & 14.79 & 26.61 \\
\hline
\end{tabular}

Finally in Table 4.6 we present additional speedup results that complement those of Table 4.2 and Table 4.5 for $n=32768$ when all 32 processors of the cluster are utilized. We obtain results that confirm the scalability of the proposed algorithm and the slight advantage of using BSPlib over LAM-MPI.

We also tested the suitability of the implementation of BSP_EFDM in a multicore environment. We used BSPlib with interprocessor communication performed through shared memory using SHMEM_SYSV primitives as these are available in BSPlib. For MPI, we used Open MPI 1.2.7. In addition, we modified our code to run under SWARM, a programming framework for multi-core processors. In these experiments we used $n=8192,16384,32768$, and $b=65,129,257,513,1025,2049,4097$. 
TABLE 4.5

Speedup results using LAM-MPI on an SMP cluster.

\begin{tabular}{rrrrrrrrrr}
\hline \multicolumn{4}{c}{$n=8192$} & \multicolumn{3}{c}{$n=16384$} & \multicolumn{3}{c}{$n=32768$} \\
\hline \multicolumn{4}{c}{$p=1: T=21.93$} & \multicolumn{3}{c}{$p=1: T=130.44$} & \multicolumn{3}{c}{$p=1: T=589.61$} \\
\hline \multicolumn{4}{c}{ Speedup } & \multicolumn{3}{c}{ Speedup } & \multicolumn{3}{c}{ Speedup } \\
\hline$b$ & $p=4$ & $p=8$ & $p=16$ & $p=4$ & $p=8$ & $p=16$ & $p=4$ & $p=8$ & $p=16$ \\
\hline 65 & 4.16 & 5.67 & 4.81 & 6.58 & 10.50 & 11.28 & 5.77 & 12.18 & 18.01 \\
\hline 129 & 4.39 & 6.75 & 7.41 & 6.74 & 11.75 & 15.10 & 5.86 & 13.02 & 22.05 \\
\hline 257 & 4.52 & 7.61 & 10.11 & 6.83 & 12.03 & 18.35 & 5.86 & 14.02 & 25.10 \\
\hline 513 & 4.55 & 8.00 & 11.66 & 6.84 & 12.88 & 21.04 & 5.82 & 14.72 & 26.92 \\
\hline 1025 & 4.51 & 7.41 & - & 6.80 & 12.76 & 22.03 & 6.03 & 15.22 & 27.62 \\
\hline
\end{tabular}

TABLE 4.6

Additional Speedup results using BSPlib and LAM-MPI on the 32-processor SMP cluster.

\begin{tabular}{rrrr}
\hline \multicolumn{2}{c}{$n=32768$} & BSPlib & LAM-MPI \\
\hline & Time $($ secs $)$ & Speedup & Speedup \\
\hline$b$ & $p=1$ & $p=32$ & $p=32$ \\
\hline 65 & 589.61 & 29.02 & 12.34 \\
\hline 129 & 589.61 & 34.72 & 19.36 \\
\hline 257 & 589.61 & 36.87 & 27.46 \\
\hline 513 & 589.61 & 40.88 & 34.74 \\
\hline 1025 & 589.61 & 40.94 & 39.86 \\
\hline
\end{tabular}

The results obtained are reported in Table 4.7. For $n=8192$ there are no noticeable differences among the three frameworks. For $n=16384$ and $n=32768$, Open MPI gives slower results. SWARM seems to be marginally faster than BSPlib for $n=16384$ and for $n=32768$ BSPlib has a marginal advantage. Both SWARM and $B S P l i b$ witness increasing efficiency in the range of $70-95 \%$, whereas efficiency for Open MPI is limited to $70-73 \%$.

5. Conclusion. We have shown how parallel computations such as those involved in American-style option valuations can be performed in parallel using the explicit finite difference method. We analyzed the proposed parallel algorithm in an architecture independent setting using the tools provided by the BSP model of computation. In addition we used the BSP programming paradigm to implement the parallel algorithm and verified its theoretical performance by carrying out an experimental study on a moderate size high latency parallel system and with a variety of parallel communication libraries. The experimental results obtained seem to confirm the conclusions drawn from the architecture independent analysis of the theoretical performance of the algorithm. In addition we have established that the proposed algorithm and its implementation have potential in a multi-core environment as well. The source code of the implementations is publically available [9]. Support in part by NSF/MRI NSF-9977508 and NSF/ITR IIS-0324816 grants is gratefully acknowledged. 
TABLE 4.7

Dual-core timing results for BSPlib (BSP), Open MPI (MPI), and SWARM.

\begin{tabular}{rrrrrrrrrr}
\hline \multicolumn{10}{c}{ Time (secs) } \\
\hline \multicolumn{3}{c}{$n=8192$} & \multicolumn{3}{c}{$n=16384$} & \multicolumn{3}{c}{$n=32768$} \\
\hline \multicolumn{4}{c}{$p=1: T=2.310$ secs } & \multicolumn{2}{c}{$p=1: T=9.118$} & \multicolumn{2}{c}{$p=1: T=37.840$} \\
\hline$b$ & BSP & MPI & SWARM & BSP & MPI & SWARM & BSP & MPI & SWARM \\
\hline 65 & 1.67 & 1.67 & 1.67 & 4.91 & 4.91 & 6.56 & 20.25 & 19.86 & 25.99 \\
\hline 129 & 1.66 & 1.67 & 1.67 & 4.91 & 4.91 & 6.54 & 20.08 & 19.75 & 25.95 \\
\hline 257 & 1.66 & 1.67 & 1.67 & 4.90 & 4.91 & 6.54 & 20.14 & 19.98 & 25.91 \\
\hline 513 & 1.66 & 1.67 & 1.66 & 4.89 & 4.90 & 6.54 & 19.98 & 19.73 & 26.08 \\
\hline 1025 & 1.65 & 1.66 & 1.66 & 4.87 & 4.91 & 6.52 & 20.01 & 19.74 & 25.99 \\
\hline 2049 & 1.66 & 1.69 & 1.68 & 4.95 & 4.97 & 6.51 & 19.96 & 19.64 & 25.91 \\
\hline 4097 & 1.88 & 1.90 & 1.88 & 5.15 & 5.18 & 6.58 & 19.96 & 19.84 & 25.84 \\
\hline
\end{tabular}

\section{REFERENCES}

[1] D.A. Bader And K. Madduri, SWARM: A Parallel Programming Framework for Multi-Core Processors, First Workshop on Multithreaded Architectures and Applications (MTAAP), Long Beach, CA, March 30, 2007.

[2] D.A. Bader, K. Madduri, and R.H. Sangani, Multicore SWARM, [www http:// multicore-swarm. sourceforge.net], 2007.

[3] F. Black And M. Scholes, The pricing of options and corporate liabilities, Journal of Political Economy, 81 (1973), pp. 637-654.

[4] P. P. Boyle, Option valuation using a three jump process, International Options Journal, 3 (1986), pp. 7-12.

[5] L. Clewlow and C. Strickland, Implementing derivative models, John Wiley \& Sons, 1998.

[6] A. V. Gerbessiotis and L. G. Valiant, Direct bulk-synchronous parallel algorithms, Journal of Parallel and Distributed Computing, 22(1994), pp. 251-267, 1994.

[7] A. V. Gerbessiotis, Architecture independent parallel binomial tree option price valuations, Parallel Computing journal, 30:2(2004), pages 303-318, Elsevier Science, BV.

[8] A. V. Gerbessiotis, Trinomial-tree based parallel option price valuations, Parallel Algorithms and Applications, Vol 18, Number 3, December 2003, pp. 181-196, Elsevier Science BV.

[9] A. V. Gerbessiotis, [www http://www.cs.njit.edu/ alexg/cluster/efdm.html], July 2007.

[10] M. W. Goudreau, J. M. D. Hill, K. Lang, W. F. McColl, S. D. Rao, D. C. Stefanescu, T. Suel, And T. Tsantilas, A proposal for a BSP Worldwide standard, BSP Worldwide, [www http://www.bsp-worldwide.org/], April 1996.

[11] R.L. Graham, T.S. Woodall, J.M. Squyres, Open MPI: A Flexible High Performance MPI, Proceedings, 6th Annual International Conference on Parallel Processing and Applied Mathematics, September 2005, Poznan, Poland, Springer Verlag Lecture Series in Computer Science, Springer.

[12] E. G. HAUG, The complete guide to option pricing formulas, McGraw-Hill, 1998.

[13] A. Ghuloum, G. Wu, X. Zhou, P. Guo, And J. Fang, Programming Option Pricing Financial Models with Qt, [www http://techresearch. intel.com/userfiles/en-us/File/terascale/Ct-appnote-option-pricing.pdf].

[14] D. B. Skillicorn, J. M. D. Hill, And W. F. MCColl, Questions and answers about BSP, Scientific Programming, 6 (1997), pp. 249-274.

[15] LAM/MPI PARAllel COMPuting, [www http://www.lam-mpi.org].

[16] E. S. SCHWARTZ, The valuation of warrants: implementing a new approach, Journal of Financial Economics, 4(1977), pp. 79-94.

[17] W. F. Sharpe, Investments, Prentice-Hall, Englewood Cliffs, New Jersey, 1978.

[18] L. G. Valiant, A bridging model for parallel computation, Communications of the ACM, 33(8), pp. 103-111, August 1990. 